\title{
Digital holographic particle image velocimetry: eliminating a sign-ambiguity error and a bias error from the measured particle field displacement
}

\author{
Thomas Ooms $^{1}$, Wouter Koek ${ }^{2}$ and Jerry Westerweel ${ }^{1}$ \\ ${ }^{1}$ Laboratory for Aero- and Hydrodynamics, Faculty of Mechanical, Maritime and Materials Engineering, \\ Delft University of Technology, Leeghwaterstraat 21, 2628 CA Delft, The Netherlands \\ ${ }^{2}$ Optics Research Group, Faculty of Applied Sciences, Delft University of Technology, Lorentzweg 1 , \\ 2628 CJ Delft, The Netherlands \\ E-mail: t.a.ooms@3me.tudelft.nl
}

Received 17 August 2007, in final form 7 December 2007

Published 23 May 2008

Online at stacks.iop.org/MST/19/074003

\begin{abstract}
In a typical digital holographic PIV recording set-up, the reference beam and the object beam propagate towards the recording device along parallel axes. Consequently, in a reconstructed volume, the real image of the recorded particle field and the speckle pattern that originates from the virtual image of the recorded particle field overlap. If the recorded particle field experiences a longitudinal displacement between two recordings and if the two reconstructed complex-amplitude fields are analysed with a 3D correlation analysis, two separate peaks appear in the resulting correlation-coefficient volume. The two peaks are located at opposite longitudinal positions. One peak is related to the displacement of the real image and the other peak is related to the displacement of the speckle pattern that originates from the virtual image. Because both peaks have a comparable height, a sign ambiguity appears in the longitudinal component of the measured particle field displacement. Additionally, the measured longitudinal particle field displacement suffers from a bias error. The sign ambiguity and the bias error can be suppressed by applying a threshold operation to the reconstructed amplitude. The sign ambiguity, characterized by $\Gamma$, is suppressed by more than a factor of 60 . The dimensionless bias error is reduced by a factor of 5 .
\end{abstract}

Keywords: digital holography, digital holographic particle image velocimetry, real image, virtual image, three-dimensional correlation, 3D correlation, HPIV, DHPIV

\section{Introduction}

Digital holographic particle image velocimetry (DHPIV) aims to perform three-dimensional (3D) flow measurements by successively measuring 3D positions of suspended tracer particles. This method quickly developed after Adams, Kreis and Jüptner [1] and Murata and Yasuda [2] first described digital holographic recordings of a particle field.

In DHPIV, a particle field is recorded in a hologram, which is subsequently reconstructed as a three-dimensional (3D) image. Such an image generally contains a real image of the recorded particle field and a speckle pattern that originates from the virtual image of the recorded particle field. A digital hologram is generally recorded with an on-axis set-up, where the object beam and reference beam approach the recording device along the same (optical) axis [3-6]. Consequently, the real image and the speckle pattern that originates from the virtual image spatially overlap [7]. When the object (particle field) moves along the longitudinal (optical) axis between two consecutive recordings, the displacement of the real image and the displacement of the speckle pattern that originates from the virtual image are 


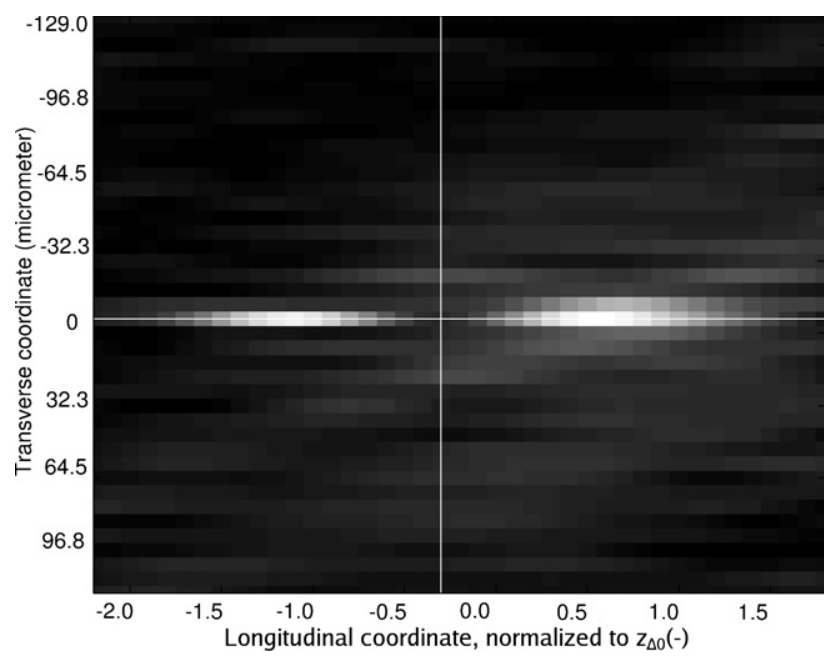

Figure 1. A slice through a typical correlation volume of an on-axis, experimentally recorded particle field that was translated $1 \mathrm{~mm}\left(=z_{\Delta 0}\right)$. The grey scale represents the correlation coefficient. The left peak is caused by the speckle pattern of the virtual image and the right peak is caused by the real image. The longitudinal position of the peaks is biased towards a smaller absolute value.

opposite in the longitudinal direction. This effect is further explained in section 2. The two calculated reconstructed complex-amplitude fields are further analysed by splitting them into interrogation volumes. These are further processed with a 3D correlation analysis, which leads to 3D correlation volumes. The opposite longitudinal displacement of the real image and the speckle pattern of the virtual image leads to the appearance of two peaks in the correlation volume. The two peaks are located along the same longitudinal line in the correlation volume and the height (correlation coefficient) and the shape of the two peaks are comparable. This is illustrated in figure 1. The presence of two peaks in the correlation volume leads to a sign-ambiguity error in the longitudinal component of the measured particle field displacement. Additionally, the speckle pattern that originates from the virtual image causes a bias error in the longitudinal component of the measured particle field displacement.

Although these errors are clearest in the case of a uniform flow in the longitudinal direction, they are also clearly present in a DHPIV-measurement result of a more realistic, nonuniform flow. A non-uniform flow is characterized by spatial gradients of the flow displacement. It is shown in section 3 that even when these spatial gradients have relatively high values (but within the generally considered limits of particle image velocimetry (PIV) [8]), the sign-ambiguity error and the bias error remain significant. In other words, without effective measures, the sign-ambiguity error and the bias error will generally significantly affect DHPIV-measurement results.

A theoretical analysis of the double correlation peak and the sign ambiguity is given in section 2 . These effects are further analysed by numerical simulations in section 3 . Section 4 shows the appearance of the predicted effects in experimental results. A method to suppress the sign-ambiguity error and the bias error is presented in section 5. It is shown that applying a threshold operation to the reconstructed particle

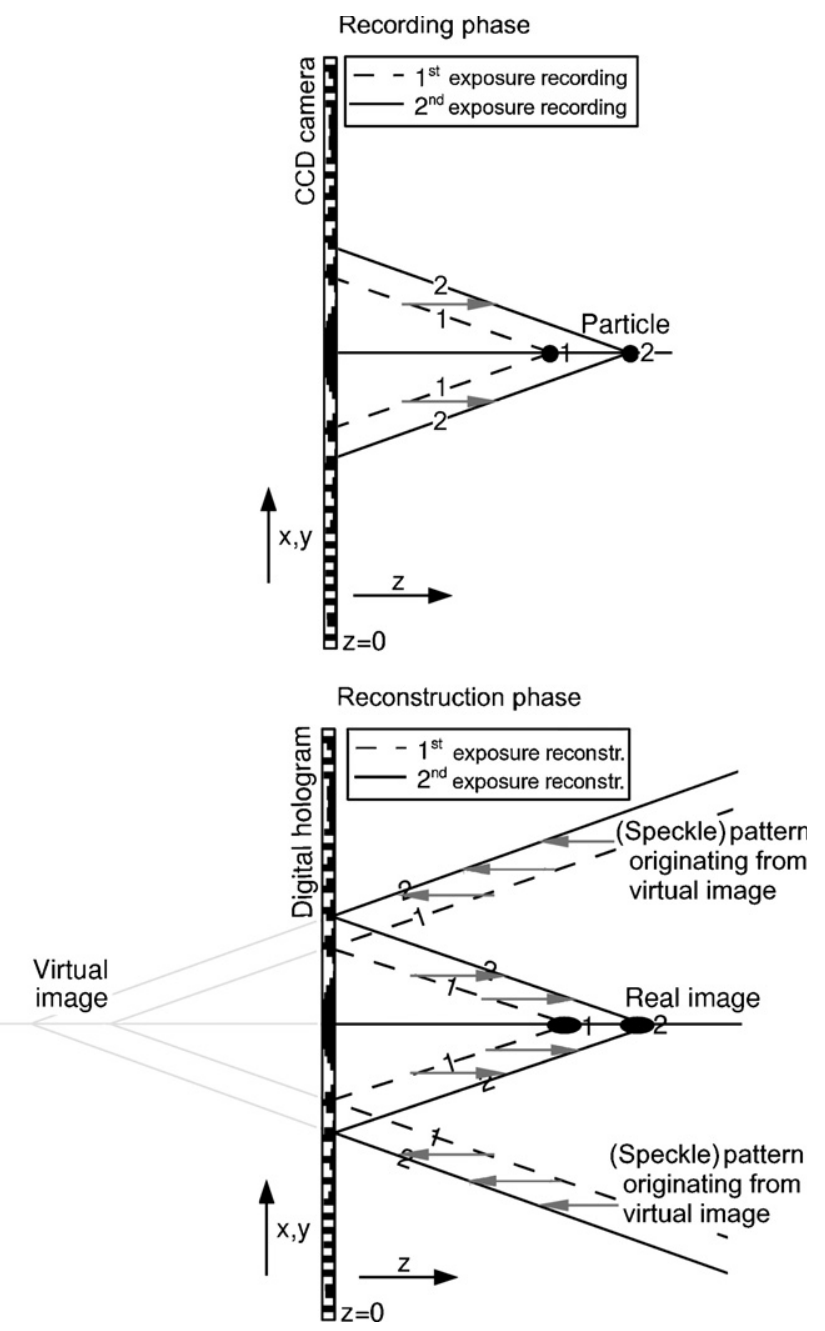

Figure 2. A particle experiences a longitudinal displacement between two recording exposures (upper figure). The reconstructed real particle image and the reconstructed (speckle) pattern of the virtual particle image show opposite longitudinal displacement between the two reconstructions (lower figure).

field image removes the sign-ambiguity error and suppresses the bias error.

Some additional clarity is given about the terminology used: in this work, the term 'virtual image' refers to the apparent origin of the reconstructed typically diverging waves, which is located in the negative $z$ domain (figure 2, lower half). This origin falls outside the reconstructed region. In this work, the term 'speckle pattern of the virtual image' or '(speckle) pattern that originates from the virtual image' refers to the waves that propagate from the virtual image and are located in the positive $z$ domain (figure 2, lower half). They fall inside the reconstructed region.

\section{Theoretical analysis}

When performing holographic PIV with a high-resolution recording medium such as a silver-halide film [9-12] or a bacteriorhodopsin film [13], the optical system allows for 
the use of an off-axis Leith-Upatnieks configuration [7, 14]. In this configuration, the reconstructed pattern that originates from the virtual image is spatially separated from the reconstructed real image and therefore does not disturb the analysis of the real image. However, in digital holographic $\mathrm{PIV}$, holograms are recorded on a CCD or CMOS image sensor, which has a relatively large pixel spacing $(4-10 \mu \mathrm{m})$ compared to the wavelength of the recorded light. This requires that the angle between the object beam and the reference beam is small so that the interference fringes are sufficiently far separated in the hologram [7] to fulfil the Nyquist sampling criterion. A DHPIV recording configuration is therefore generally an on-axis configuration where the object beam and reference beam propagate towards the CCD chip along the same optical axis. In the numerical reconstruction of the hologram, the real image and the pattern that originates from the virtual image are located in the same spatial region. The zeroth order is eliminated during hologram reconstruction [15]. Consequences of the overlap of the real image and the speckle pattern that originates from the virtual image on measurement results are described in this section.

To understand the effect of the speckle pattern of the virtual image on DHPIV-measurement results, a singleparticle object is considered (figure 2). The particle is recorded in two time-separated holograms (double-frame, single-exposure [16]). The two hologram reconstructions each contain a real image and a pattern that originates from the virtual image. The hologram plane, or CCD plane, is located at $z=0$. The recorded particle, the reconstructed real image and the reconstructed speckle pattern that originates from the virtual image are located at positive $z$-values. When the particle moves a distance $z_{\Delta 0}$ away from the CCD plane between the two recordings, or from $\left(x_{p}, y_{p}, z_{p}\right)$ to $\left(x_{p}, y_{p}, z_{p}+z_{\Delta 0}\right)$, the focus point of the real particle image moves from $\left(x_{p}, y_{p}, z_{p}\right)$ to $\left(x_{p}, y_{p}, z_{p}\right.$ $\left.+z_{\Delta 0}\right)$, corresponding to a shift in the positive $z$-direction. However, the reconstructed pattern that originates from the virtual image is a spherical wave that first diverges from the point $\left(x_{p}, y_{p},-z_{p}\right)$ and then from the point $\left(x_{p}, y_{p}\right.$, $\left.-z_{p}-z_{\Delta 0}\right)$, corresponding to a shift in the negative $z$-direction. In other words, there are two superimposed reconstructed fields that move in opposite longitudinal directions. When the object consists of several particles that are moved uniformly, the effect is the same: when the object is moved uniformly in the positive $z$-direction, the reconstructed real particle images move uniformly in the positive $z$-direction. The reconstructed field that originates from the virtual image forms a 3D speckle pattern because the particle images function as a large set of random phase scatterers that interfere coherently [7]. When the particle fields is moved uniformly, it is expected that the speckle pattern keeps the same profile because the optical path lengths between the scatterers remain unchanged. Similar to the single-particle case, when the recorded particle field moves uniformly in the positive $z$-direction, the speckle pattern that originates from the virtual image moves uniformly in the negative $z$-direction. When the reconstructed particle fields of the first and second recordings are analysed by a $3 \mathrm{D}$ correlation analysis, two peaks appear in a typical correlation volume, one corresponding to the positive $z$-shift of the real image (named: real-image peak) and the other corresponding to the negative $z$-shift of the speckle pattern that originates from the virtual image (named: virtual-image peak). The vector $\left(x_{\Delta 0}, y_{\Delta 0}, z_{\Delta 0}\right)$ is defined as the real, physical displacement of the recorded particle field. With a recorded particle field displacement $\left(x_{\Delta 0}, y_{\Delta 0}, z_{\Delta 0}\right)$, the real-image peak is expected at $\left(x_{\Delta 0}, y_{\Delta 0},+z_{\Delta 0}\right)$ and the virtual-image peak is expected at $\left(x_{\Delta 0}, y_{\Delta 0},-z_{\Delta 0}\right)$. Further, the vector $\left(x_{\Delta m}, y_{\Delta m}, z_{\Delta m}\right)$ is defined to indicate the measured particle field displacement.

In this study, the 3D correlation analysis is based on the reconstructed complex amplitude, not on the reconstructed real intensity. Coupland and Halliwell [17] showed that the use of the complex amplitude leads to a more robust correlation analysis. With this choice, the height of the real-image peak and the virtual-image peak can be calculated. The height (correlation coefficient) of the virtual-image peak, $r\left(x_{\Delta 0}, y_{\Delta 0}\right.$, $\left.-z_{\Delta 0}\right)$, and the real-image peak, $r\left(x_{\Delta 0}, y_{\Delta 0},+z_{\Delta 0}\right)$, of the crosscorrelation analysis are equal if the autocovariance function of the speckle pattern that originates from the virtual image, $C_{v}$, and the autocovariance function of the real image, $C_{r}$, at their origins $\left(C_{v}(0,0,0)\right.$ and $\left.C_{r}(0,0,0)\right)$ are equal [18]. They are defined as [18]

$$
\begin{aligned}
& C_{v}(0,0,0) \\
& =\left(V_{o}^{-1} \iiint\left|u_{v}\right|^{2} \mathrm{~d} V\right)-\left|V_{o}^{-1} \iiint u_{v} \mathrm{~d} V\right|^{2} \\
& C_{r}(0,0,0) \\
& =\left(V_{o}^{-1} \iiint\left|u_{r}\right|^{2} \mathrm{~d} V\right)-\left|V_{o}^{-1} \iiint u_{r} \mathrm{~d} V\right|^{2}
\end{aligned}
$$

where $V_{0}=\iiint \mathrm{d} V, u$ is a complex amplitude as defined by Goodman [6], $u_{v}$ is the complex amplitude of the speckle pattern of the virtual image, $u_{r}$ is the complex amplitude of the real image and $V$ represents a volume variable. The spatial average of the complex light amplitude of a light wave is generally zero $\left(\iiint u_{v} \mathrm{~d} V=0\right.$ and $\left.\iiint u_{r} \mathrm{~d} V=0\right)$ and the second term on the right-hand side of equations (1) and (2) can therefore be neglected. Like $C_{v}(0,0,0)$ and $C_{r}(0,0,0)$, the light energy of the speckle pattern that originates from the virtual image and the energy of the real image in the reconstruction volume $\left(E_{v}\right.$ and $\left.E_{r}\right)$ are proportional to the volume integral of the absolute square of the complex light amplitude of the speckle pattern of the virtual image and the real image, respectively [7],

$$
\begin{aligned}
& E_{v}=c^{-1} \iiint\left|u_{v}\right|^{2} \mathrm{~d} V \\
& E_{r}=c^{-1} \iiint\left|u_{r}\right|^{2} \mathrm{~d} V
\end{aligned}
$$

where $c$ is the speed of light. Holographic reconstruction is based on the principle of diffraction. The speckle patterns of the virtual image and the real image are formed by the $+1 \mathrm{st}$ and -1 st diffraction orders. Theory describes that the energies in both orders are equal [7]. The energies of the speckle pattern that originates from the virtual image and the real 
image remain equal in any reconstruction plane parallel to the hologram plane. In a reconstruction plane at a finite distance from the hologram, the speckle pattern that originates from the virtual image is, at first sight, expected to diverge out of the reconstruction region. However, when the size of the reconstruction plane matches the size of the recorded hologram and when the reconstruction is performed with a fast-Fourier-transform (FFT) algorithm (section 3), the speckle pattern that originates from the virtual image is kept inside the reconstruction region because the image is 'folded' back into the reconstruction region [19]. Because the speckle patterns of the virtual image and the real image are kept inside the reconstruction region, the energy of the speckle pattern of the virtual image is equal to the energy of the real image at any distance from the hologram. The relation $E_{v}=E_{r}$ and equations (1)-(4) show that the heights (cross-correlation coefficient) of the real-image peak and the virtual-image peak are generally equal or

$$
r\left(x_{\Delta 0}, y_{\Delta 0},-z_{\Delta 0}\right)=r\left(x_{\Delta 0}, y_{\Delta 0},+z_{\Delta 0}\right) .
$$

Generally, holographic PIV algorithms extract the local particle field displacement from a 3D correlation volume by finding the position of the maximum value of the correlation volume (peak position). For an appropriate measurement of the local particle field displacement, the position of the realimage peak should be found [9-12]. Because both correlation peaks have a similar height, it is unpredictable whether the 'peak-finding' algorithm will 'find' the position of the virtualimage peak or the real-image peak. This leads to a sign ambiguity in the longitudinal component of the displacement vector.

The sign ambiguity of the measured particle field displacement occurs only in the longitudinal direction and not in the transverse direction. If a particle field is moved over a transverse distance $\left(+x_{\Delta 0},+y_{\Delta 0}, 0\right)$, both the real image and the speckle pattern that originates from the virtual image move in the positive $\left(+x_{\Delta 0},+y_{\Delta 0}, 0\right)$ direction. When the two reconstructed particle fields are analysed by a 3D correlation analysis, the real-image peak and the virtual-image peak coincide at $\left(+x_{\Delta 0},+y_{\Delta 0}, 0\right)$.

For clarity, the sign ambiguity that is discussed in this paragraph is absolutely not related to the directional ambiguity that follows from a PIV autocorrelation analysis where a sign ambiguity exists for all components of the displacement vector [20].

Additionally, figure 1 reveals that the real-image (right) peak is not located at exactly $\left(0,0, z_{\Delta 0}\right)$ but is shifted towards the origin. This illustrated the bias error in $z_{\Delta m}$. This error is caused by the speckle pattern that originates from the virtual image in the analysed volume, as explained in appendix A.

\section{Numerical simulation}

In this section, the appearance of two peaks in a typical correlation volume and the sign ambiguity of $z_{\Delta m}$ are illustrated with a numerical simulation. A description of the simulation procedure is followed by the simulation results.

An object volume of $N_{x} \times N_{y} \times N_{z}=1024 \times 1024 \times$ 200 pixels is defined. The transverse pixel spacing is $6.45 \mu \mathrm{m}$ and the longitudinal pixel spacing is $100 \mu \mathrm{m}$. A particle field is located $10-30 \mathrm{~mm}$ from the hologram. These values match the parameters of our experimental set-up and procedure (section 4), which allows for comparison of numerical and experimental results. One thousand particles are randomly distributed in this volume by setting the value of a pixel in the object volume to 1 . With this method, the particles are modelled as point sources. When a particle does not have an integer-pixel location, the value 1 is distributed over the nearest eight pixels so that the centre of gravity of the eight pixel values matches the position of the particle. Light scattering from the particles and propagation to the digital hologram are simulated by convoluting each object plane $O_{z}(x, y)$ by a Rayleigh-Sommerfeld point-source diffraction term $K_{z}(x, y)$ [7]. The complex amplitude of the scattered object light at the hologram $U_{0}(x, y)$ is the sum of the contributions of all object planes:

$$
U_{0}(x, y) \propto \sum_{z}\left(O_{z}(x, y) * K_{z}(x, y)\right)
$$

where $*$ represents a $2 \mathrm{D}$ convolution and

$$
K_{z}(x, y)=\frac{1}{\mathrm{i} \cdot \lambda} \frac{\exp \left(\mathrm{i} \cdot k \cdot \sqrt{x^{2}+y^{2}+z^{2}}\right)}{\sqrt{x^{2}+y^{2}+z^{2}}}
$$

where $\mathrm{i}$ is the imaginary unit, $\lambda$ is the wavelength, $k$ is the wavenumber, $x$ and $y$ are the transverse coordinates and $z$ equals the distance from the object plane to the parallel digital hologram. The cosine term in the Rayleigh-Sommerfeld point-source diffraction term can be neglected for this low-NA set-up. The term $K_{z}(x, y)$ is set to zero at the $(x, y)$ positions where the oscillations of $K_{z}(x, y)$ violate the Nyquist sampling criterion $[7]^{3}$. At the hologram, the complex amplitude of the object light is coherently added to a plane-wave reference beam, $R(x, y)$. The absolute value of this sum is squared to obtain the hologram intensity, $I_{h}(x, y)$ :

$$
I_{h}(x, y)=\left|R(x, y)+U_{0}(x, y)\right|^{2} .
$$

The size of the hologram matches the size of the recorded object $\left(1024 \times 1024\right.$ pixels, $\left.6.6 \times 6.6 \mathrm{~mm}^{2}\right)$. A second hologram is recorded after a 3D particle field displacement. In this simulation, the grey level quantization of the hologram is not reduced to typical experimental values (i.e. of a CCD camera). Work by Westerweel [22] has shown that grey level quantization has no significant influence on a typical PIV correlation analysis. This argument is expected to be also valid for a $3 \mathrm{D}$ correlation analysis and omitting quantization in this simulation should have no influence on final results.

Reconstruction of the two holograms is performed similarly. The hologram is virtually exposed to a plane-wave reconstruction beam, and the hologram intensity, $I_{h}(x, y)$, is now regarded as a spatially varying amplitude transmittance function, $t(x, y)$. The light amplitude in a reconstruction plane, $U_{z}(x, y)$, is calculated by convoluting the hologram intensity with $K_{z}(x, y)$ :

$$
U_{z} \propto t(x, y) * K_{z}(x, y) .
$$

3 Coupland [21] suggests that recording undersampled digital holograms can lead to successful reconstructions. However, in this work the maximal recorded signal frequency is chosen at the Nyquist sampling frequency. This avoids the appearance of the so-called ghost images in the reconstruction. This approach seems most suitable in this case because the displacement of the particle images can be precisely controlled. 
The spatial region of the reconstruction matches the spatial region of the hologram.

The two reconstructed volumes are further analysed to extract local 3D displacement vectors. In other papers, various correlation methods have been described to calculate 3D displacement. One approach calculates two non-parallel 3D-2C (two vector components) vector fields from a 3D reconstruction by a conventional $2 \mathrm{D}$ PIV analysis. The two $3 \mathrm{D}-2 \mathrm{C}$ vector fields are then geometrically combined to one $3 \mathrm{D}-3 \mathrm{C}$ vector field $[9,11]$. Another approach uses the socalled digital shearing method [23]. Herrmann and Hinsch calculate 3D displacement vectors with a 3D correlation analysis [24]. We choose to use the same method because of the straightforward nature of this method. While Hermann and Hinsch use intensity data for their correlation, we use the reconstructed complex amplitude. Both approaches are valid because the correlation operation is defined for real data, as well as for complex data [18]. It should be noted that Meng et al [25] critically discuss the suitability of a 3D correlation analysis for holographic PIV. They discuss the effect of speckle noise in the reconstructed image on a 3D correlation analysis and the effect of the longitudinally elongated particle images on a $3 \mathrm{D}$ correlation analysis [25]. However, it is shown in section 5 that the effect of speckle noise on a $3 \mathrm{D}$ correlation can be effectively suppressed. It is further expected that the relatively large longitudinal size of the particle images has no significant deteriorating effect on 3D correlations in this case, because the longitudinal size of an interrogation volume is larger than the typical longitudinal size of the particle images ${ }^{4}$. Then, for further data processing, the two 3D reconstruction volumes are each split into interrogation volumes. The size of an interrogation volume is 128 pixels in the transverse direction and 32 pixels in the longitudinal direction. With the transverse pixel size of $6.45 \mu \mathrm{m}$ and a longitudinal pixel size of $100 \mu \mathrm{m}$, the volume of one interrogation volume is $0.83 \times 0.83 \times$ $3.2 \mathrm{~mm}^{3}=2.2 \mathrm{~mm}^{3}$ and an interrogation volume is expected to contain, on average, 2-3 particles. Although, in practice, the presence of only two to three tracer particles in an interrogation volume could lead to relatively many invalid displacement measurements [8], we expect that the a priori knowledge of the particle displacement in these simulations makes the seeding density acceptable. The interrogation-volume overlap is $50 \%$ in all three directions. The interrogation-volume dimensions are obtained by considering that a larger interrogation volume would lead to large demands on computer processing speed (CPU) and the computer memory (RAM). Much smaller interrogation volumes could lead to relatively many invalid displacement measurements because an interrogation volume would contain, on average, too few real particle images. The corresponding interrogation volumes of both holograms are processed comparably to conventional 2D digital PIV [16]: the average amplitude of the two interrogation volumes is subtracted and both volumes are zero-padded to a size of

4 The longitudinal size of the interrogation volume is chosen at $3.2 \mathrm{~mm}$. The particle image depth-of-focus (longitudinal size of particle image) is less than $1 \mathrm{~mm}$ in the described simulations, and about equal to $1 \mathrm{~mm}$ in the experiments [6].
$256 \times 256 \times 64$ pixels. The correlation is defined by Shanmugan and Breipohl [18] as

$$
r_{12}(\vec{x})=\frac{\left|E\left(\tilde{U}_{1}^{*}(\vec{\xi}) \cdot \tilde{U}_{2}(\vec{\xi}+\vec{x})\right)\right|}{\sqrt{E\left(\tilde{U}_{1}^{*}(\vec{\xi}) \cdot \tilde{U}_{1}(\vec{\xi})\right) \cdot E\left(\tilde{U}_{2}^{*}(\vec{\xi}) \cdot \tilde{U}_{2}(\vec{\xi})\right)}}
$$

where $r_{12}(\vec{x})$ is the cross-correlation coefficient, $E$ is the expectation value (or mean), $\tilde{U}_{1}$ and $\tilde{U}_{2}$ are the complexamplitude data of interrogation volumes 1 and 2, respectively, after subtraction of the mean complex amplitude and after zero-padding, $\vec{x}$ and $\vec{\xi}$ are three-dimensional spatial coordinates and ...* is a complex conjugate. The $3 \mathrm{D}$ crosscorrelation in equation (10) is implemented with an FFT algorithm: the complex conjugate of the FFT of the data of interrogation volume 1 is multiplied by the FFT of the data of interrogation volume 2 . The absolute value of the inverse FFT of the product is calculated and the result is then divided by the square root of the variance of the data of both interrogation volumes to obtain a 3D volume of crosscorrelation coefficients.

The integer-pixel position of the global maximum of the correlation cube $\left(x_{m}, y_{m}, z_{m}\right)$ is found and the correlation cube is then divided by a 3D weighting function to compensate for the fact that each point in the correlation volume is the result of a different volume overlap between interrogation volumes 1 and 2 [16]. Then, a sub-pixel estimate of the $z$-position of the correlation peak is determined by making a sevenpoint least-squares Gaussian fit on the points between $\left(x_{m}\right.$, $\left.y_{m}, z_{m}-3\right)$ and $\left(x_{m}, y_{m}, z_{m}+3\right)$. The obtained value is the defined measured longitudinal particle field displacement or $z_{\Delta m}$. No sub-pixel estimate is made of the $x$ - and $y$-positions of the correlation peak because the accuracy of the integer-pixel position is currently sufficient for this study. The calculations are performed on a personal computer (CPU: AMD $2.5 \mathrm{GHz}$; OS: Linux Red Hat), using Matlab 7. Generating and reconstructing a hologram and performing the $3 \mathrm{D}$ correlation analysis takes about $1 \mathrm{~h}$.

In a first numerical simulation, the imposed particle field displacement is $\left(x_{\Delta}, y_{\Delta}, z_{\Delta}\right)=\left(0,0, z_{\Delta 0}\right)$ and $1443 \mathrm{D}$ correlations are performed. Figure 3 illustrates the correlation coefficient on a line through the centre of the correlation volume $\left(0,0, z_{\Delta}\right)$, averaged over the 144 correlation spots. As predicted in section 2, two peaks appear with a similar height. The longitudinal component of the 144 measured displacement vectors $\left(z_{\Delta m}\right)$ is given in the upper two histograms in figure 4 . The sign-ambiguity error and the bias error of $z_{\Delta m}$ are visible.

This paragraph discusses whether the sign-ambiguity error and the bias error are only present in the measurement results of a uniform longitudinal flow or also in the measurement result of a non-uniform flow. This question is investigated for the following reason: the speckle pattern that originates from a virtual particle image is spread over a larger transverse spatial range than a typical real particle image (figure 2). Therefore, the recorded particles that contribute to the virtual-image speckle pattern in a certain interrogation region are distributed over a wider transverse spatial range than the recorded particles that contribute to the real image in this interrogation region. Therefore, for a flow with a given spatial velocity gradient, the transverse displacement variation of the 


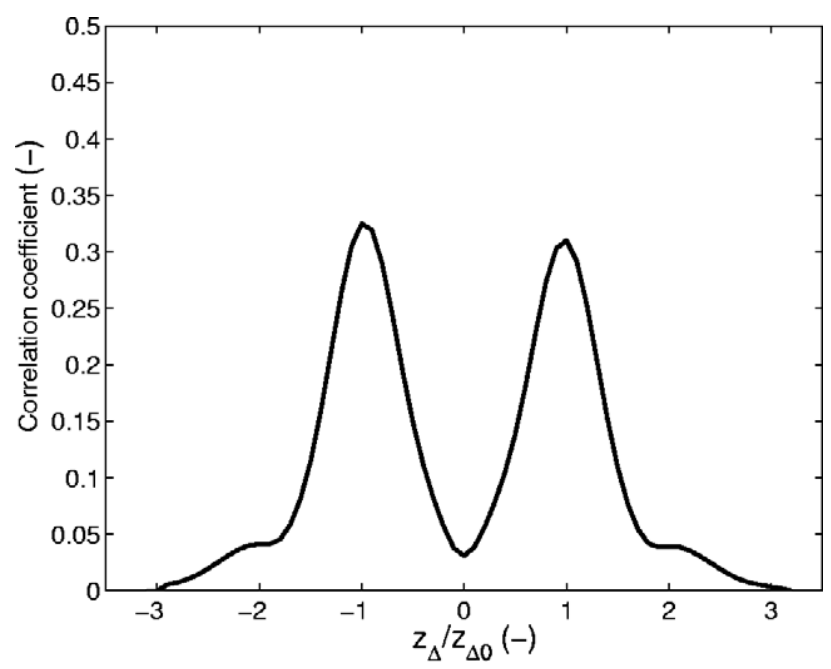

Figure 3. The correlation coefficient on a line through the centre of the correlation volume $\left(0,0, z_{\Delta} / z_{\Delta 0}\right)$, averaged over 144 correlation spots (i.e. averaging over total reconstructed volume). The left peak is caused by the speckle pattern of the virtual image and the right peak is caused by the real image. This result is calculated with a numerical simulation.

recorded particles that correspond to the virtual-image speckle pattern in the considered interrogation region is larger than the transverse displacement variation of the recorded particles that correspond to the real image in the interrogation region. Keane and Adrian [8] describe that a displacement variation of the recorded particle field lowers the corresponding correlation peak [8]. Consequently, it could be expected that the height of the virtual-image peak is on average lower than the height of the real-image peak. Therefore, when analysing DHPIV measurements of a flow with a spatial gradient, it could be possible that the real-image peak is 'detected' more often than the virtual-image peak and the sign ambiguity is reduced. A numerical simulation is performed to investigate whether this effect occurs or whether the sign-ambiguity error and the bias error are significantly present in the measurement result of a flow with displacement gradients. For this investigation, a flow is analysed that consists of a combination of a uniform displacement term along the longitudinal $z$-axis and a pure strain flow along the transverse axes (i.e. $\left(x_{\Delta}, y_{\Delta}, z_{\Delta}\right)=(\alpha x$, $\left.\left.-\alpha y, z_{\Delta 0}\right)\right)$. Here $\left(x_{\Delta}, y_{\Delta}, z_{\Delta}\right)$ is a displacement vector, $(x, y, z)$ is a position vector, $\alpha$ is a constant and $z_{\Delta 0}$ is a uniform longitudinal displacement term. For clarity, a schematic plot of this displacement field is given in figures $5(a)$ ( $x y$-plane) and 5(b) ( $x z$-plane). The simulated particle field is holographically recorded twice. The reconstructions and interrogation volumes are analysed as has been described in this section. A measured 3D particle-image displacement field is obtained. A schematic plot of an $x z$-plane of a typical measured displacement field is shown in figure $5(c)$. This figure illustrates that the transverse component of the measured displacement is accurately retrieved. However, the longitudinal component of the measured displacement appears random which illustrates the sign-ambiguity error. Histograms of the measured longitudinal particle field displacement

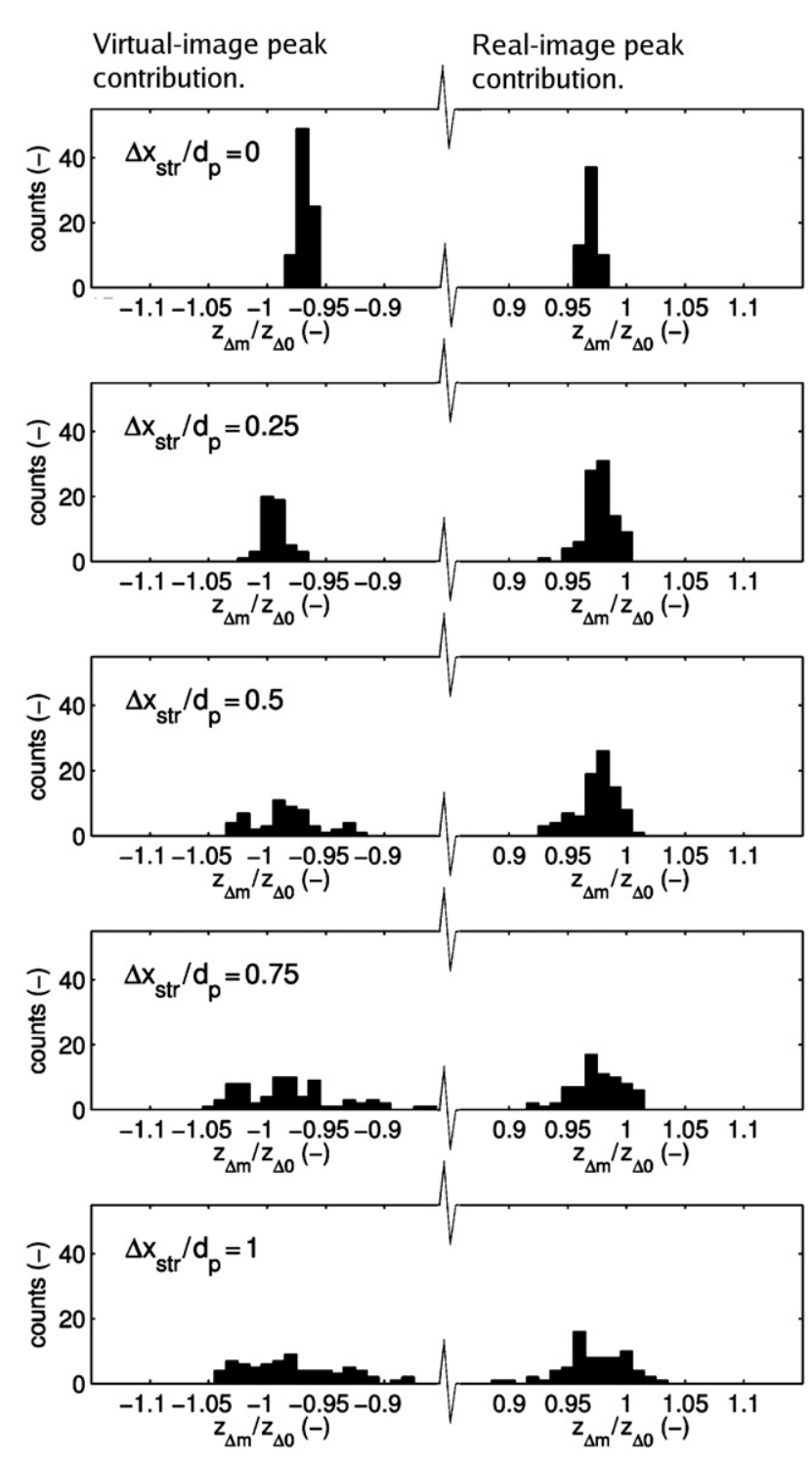

Figure 4. Histograms of the measured longitudinal component of the displacement vector $\left(z_{\Delta m}\right)$. The histogram peak in the negative range of $z_{\Delta m}$ is generated by detections of the virtual-image peak. The histogram peak in the positive range of $z_{\Delta m}$ is generated by detections of the real-image peak. From top to bottom the histograms correspond to an increasing transverse pure strain displacement. This result is calculated with a numerical simulation.

$\left(\equiv z_{\Delta m}\right)$ for a total of 144 interrogation spots are given in figure 4. The left and right columns of histograms cover respectively the relevant negative and positive range of $z_{\Delta m}$. The left column shows measured longitudinal displacements of the speckle pattern that originates from the virtual image while the right column shows measured displacements of the real image. The strain rate $(\alpha)$ increases from the top to bottom and is directly proportional to $\Delta x_{\mathrm{str}} / d_{p}$, which is named the transverse displacement variation. This is the variation of the transverse particle displacement within an interrogation volume divided by the particle-image diameter [8]. All histograms show peaks at a negative $z_{\Delta m}$ and a positive $z_{\Delta m}$, which illustrates the sign ambiguity of $z_{\Delta m}$. Note that the 

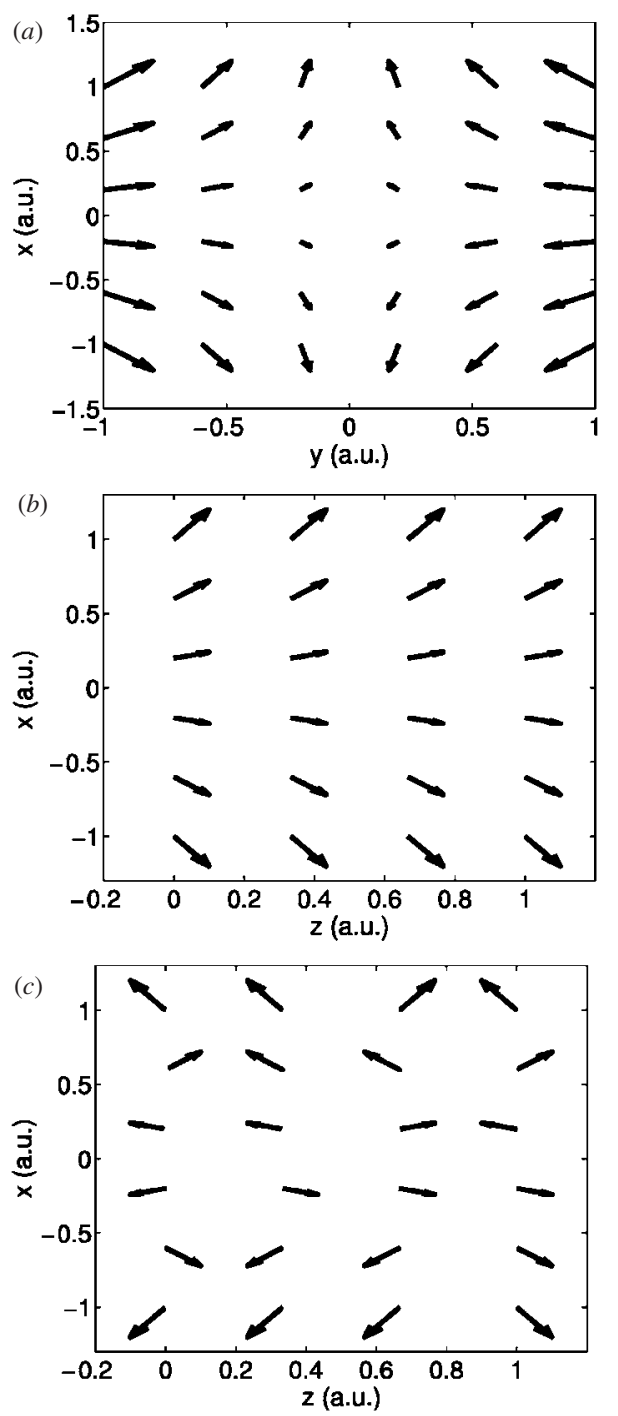

Figure 5. Schematic plots of the displacement fields: $(a)$ applied particle displacement, $x y$-view, $(b)$ applied particle displacement, $x z$-view and $(c)$ measured particle-image displacement, $x z$-view.

height of both histogram peaks decreases for an increasing strain rate. At $\left|\Delta x_{\text {str }} / d_{p}\right|=1$, the peak that corresponds to the speckle pattern of the virtual image remains. This indicates the continuing existence of the sign ambiguity. Because PIV is generally performed within the range $\left|\Delta x_{\text {str }} / d_{p}\right|<1$ [8], the sign ambiguity is expected to have a significant effect on a DHPIV 3D-correlation analysis of a general flow. Also note that the measured longitudinal displacement is, on average, not equal to $z_{\Delta m} / z_{\Delta 0}=-1.00$ and $z_{\Delta m} / z_{\Delta 0}=+1.00$, but is biased to a smaller absolute value, which indicates the described bias error.

\section{Experimental procedure}

In this section, the sign ambiguity of $z_{\Delta m}$ is illustrated experimentally. A description of the experimental set-up is followed by the experimental results. Our DHPIV set-up can

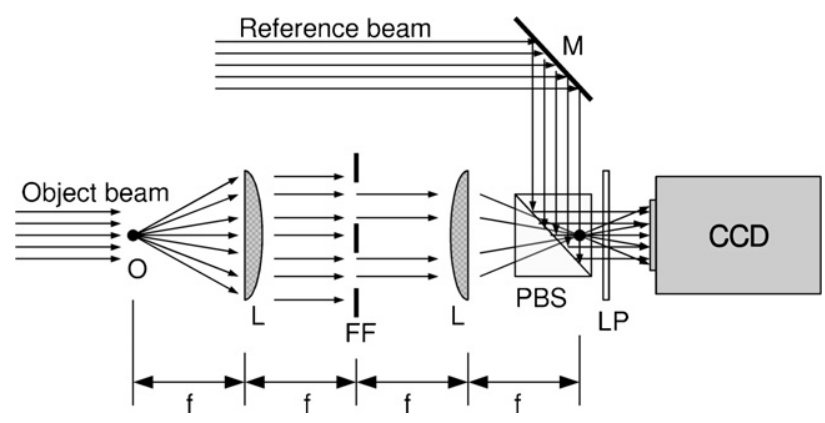

Figure 6. Optical configuration for recording a digital hologram. $\mathrm{O}$ is the recorded object, $\mathrm{L}$ is a plano-convex lens, FF is an optical Fourier filter, PBS is a polarizing beam splitter, M is a mirror, LP is a linear polarizer and $f$ is the focal length of the lenses.

be described as an in-line on-axis digital holographic recording system. It only differs from other published DHPIV systems [3-5] by initially having a spatially separated object beam and reference beam and by having a Fourier filter in the object beam [6], as shown in figure 6. The filter acts to avoid aliasing in the hologram [6], remove the unscattered light from the object beam and reduce the particle-image depth-of-focus [6]. A smaller particle-image depth-of-focus generally leads to a smaller longitudinal size of the real-image peak in a correlation volume. It is expected that this improves the accuracy of the measured longitudinal particle field displacement $\left(z_{\Delta m}\right)$. Filtering in the frequency (Fourier) domain of the hologram is not expected to have any other effect on a consequent 3D correlation analysis. The findings of this work are expected to be equally valid for a conventional in-line DHPIV system without Fourier filtering. The illumination source is a diodepumped continuous-wave $150 \mathrm{~mW}$ laser (Coherent, Compass $315 \mathrm{M}-150$ ) with a wavelength of $532 \mathrm{~nm}$. The laser beam is expanded, collimated and then split into an object beam and a reference beam to enable separate intensity control. The first beam illuminates the object, which is in our case a tank filled with resin and stationary 40-63 $\mu \mathrm{m}$ diameter Sphericel tracer particles with a density of approximately $10^{3}$ particles $\mathrm{cm}^{-3}$. The experiment makes use of the forward-scattered light. The longitudinal dimension of the tank is $20 \mathrm{~mm}$. This object with stationary particles allows for full control of the position of the particles, and hence for verification of measurement results. The scattered light goes through a positive plano-convex lens with a focal length $f=300 \mathrm{~mm}$ and a diameter of $50 \mathrm{~mm}$, through a spatial filter and through a second identical positive lens. The spatial filter consists of a concentric opaque disc with a diameter, $C$, of $10 \mathrm{~mm}$ and an aperture with a diameter, $D$, of $24 \mathrm{~mm}$. The lenses and the spatial filter are separated by the focal length of the lenses. These optical elements function as a bandpass Fourier filter. The object beam is combined with the reference beam by a beam splitter and then passes through a linear polarizer. The linear polarizer ensures uniform polarization at the CCD chip and solves possible loss of polarization due to light scattering from the object. The reference beam and the object beam propagate towards the CCD camera along the same optical axis and form a hologram on the CCD chip of a lensless digital camera (PCO Sensicam 


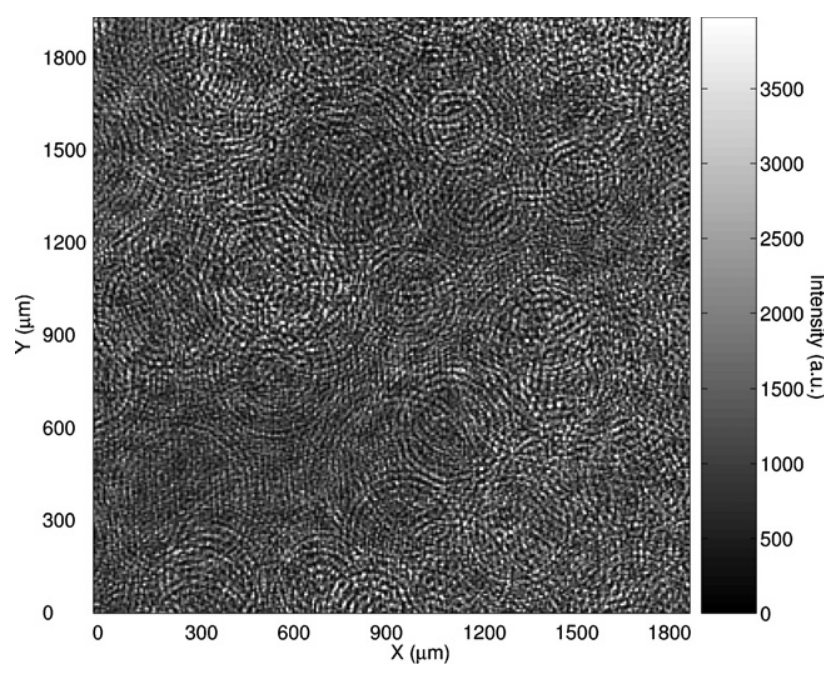

Figure 7. A part of a recorded digital hologram.

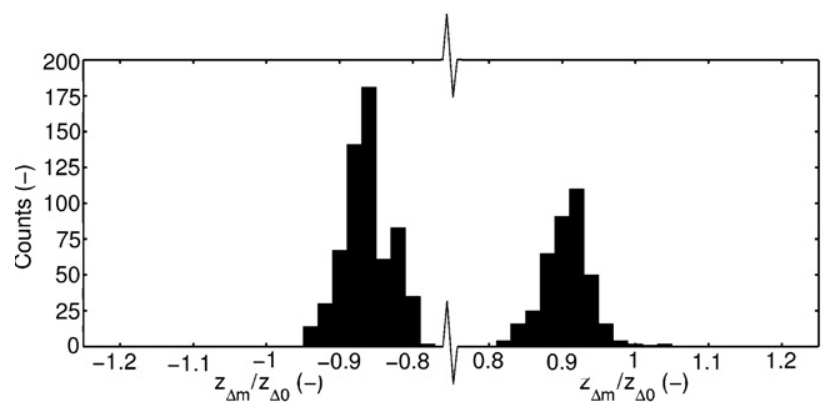

Figure 8. Histogram of the longitudinal component of the experimentally measured particle field displacement. One thousand interrogation spots are analysed. The reconstructed complex amplitude is used for the correlation analysis.

$690 \mathrm{LL})$ with $1376 \times 1040$ pixels and a pixel spacing of $6.45 \mu \mathrm{m}$. The grey level quantization of the camera is 4096 . A part of a typical recorded hologram is shown in figure 7 . The camera was positioned in a region between 10 and $30 \mathrm{~mm}$ behind the real particle field image formed by the Fourier filter. The particle field is holographically recorded, moved a distance $z_{\Delta 0}$ along the longitudinal axis and recorded again. The distance $z_{\Delta 0}$ is chosen at a suitable value of exactly $1 \mathrm{~mm}$ (error $<1 \%$ ). Reconstruction of the hologram and the 3D correlations are performed as in numerical simulations (section 3). The dimensions of a 3D reconstruction pixel and an interrogation volume are equal to the dimensions given in section 3 (pixel: $6.45 \times 6.45 \times 100 \mu \mathrm{m}^{3}$; interrogation volume: $\left.128 \times 128 \times 32 \mathrm{pix}^{3}\right)$. This measurement analyses 1000 interrogation spots.

A slice through a typical correlation volume (figure 1) clearly shows a virtual-image peak (left) and a real-image peak (right). $z_{\Delta m}$ for 1000 correlations is plotted in a histogram in figure 8 . The left and right histogram peaks are respectively generated by detection of the virtual-image peak and the realimage peak. As expected, figure 8 again illustrates the bias error of $z_{\Delta m}$. The height difference between the two peaks in figure 8 is probably caused by the relation between the seeding

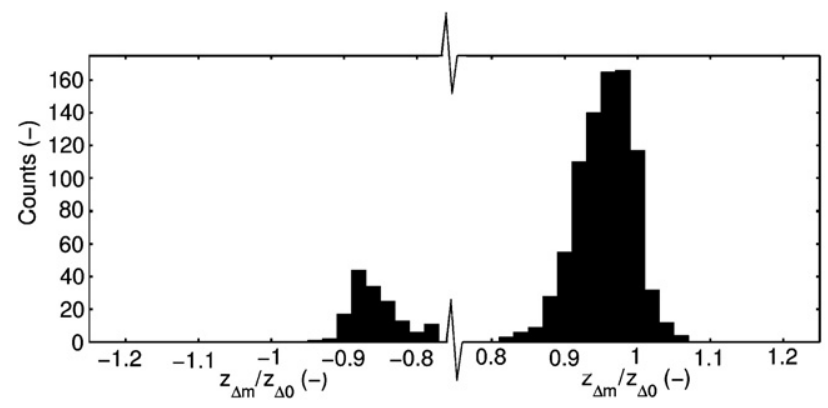

Figure 9. Histogram of the longitudinal component of the experimentally measured particle field displacement. One thousand interrogation spots are analysed. The reconstructed real intensity is used for the correlation analysis.

density and the interrogation-volume size and a possible inhomogeneity of the particle distribution in the recorded volume. If, for example, the seeding density would be very low and the interrogation volumes very small, most interrogation regions would contain only a virtual-image speckle pattern while only a few interrogation regions would contain a real particle image. As a result, the majority of the interrogations would only contribute to the virtual-image-related peak in a displacement histogram. If, for example, the recorded particles would be distributed so inhomogeneously that the real particle images are located in a small part of all interrogation regions, the majority of the interrogation regions would contain only a virtual-image speckle pattern. As a result, the majority of the interrogations would again contribute only to the virtualimage-related-peak in a displacement histogram.

\section{Suppressing the sign ambiguity and the bias error of $z_{\Delta m}$}

In this section, methods are investigated to remove or suppress the sign ambiguity and the bias error of $z_{\Delta m}$.

As said in section 2, this study uses the reconstructed complex amplitude for the 3D correlation. If, on the other hand, the reconstructed real intensity is used, the resulting signambiguity and the bias error may be different. A cause of this effect is that the real images of particles are strongly localized high-intensity images, while the speckle pattern of the virtual image of particles is a non-localized low-intensity image. If a particle field image is converted from complex amplitude to real intensity by a squaring operation, the high-intensity data (real image) is amplified more than low-intensity data (speckle pattern of the virtual image). On average, this amplifies the height of the real-image peak with respect to the height of the virtual-image peak. It is expected that the sign ambiguity and the bias error are then less pronounced. However, an experimental investigation still indicates that when the reconstructed real intensity is used for the correlation analysis, the sign ambiguity and the bias error keep a significant value: the left peak in figure 9 indicates that $15 \%$ of the measured displacements originate from the virtual-image peak, implying a significant sign ambiguity. Additionally, the peak that originates from the real-image peak (right) is located left of 
the expected position, corresponding to a bias error of $4.5 \%$. Hence, whether the reconstructed complex amplitude or the real intensity is used, a solution is desired to suppress the sign ambiguity and the bias error.

One approach to avoid the sign ambiguity and the bias error of $z_{\Delta m}$ is to avoid the presence of the speckle pattern that originates from the virtual image in the reconstruction region. Onural and Scott [26] proposed a truncated series expansion operator that numerically decodes the real image from the hologram while suppressing the speckle pattern of the virtual image. Another approach is taken by Pan and Meng [3], who identify and select the real images of particles by studying the properties of the complex reconstruction signal. These two methods, however, are only suitable for opaque particles $[3,26]$ that do not affect the phase of the light of the object beam. These methods cannot be applied successfully to transparent particles with a finite longitudinal size. Schnars and Jüptner [27] and Cuche et al [15] separated the real image of an object by using an off-axis configuration with a small angle between the object- and reference beam. However, such a method works by separating the real image from the zero order and virtual image in the spatial frequency (Fourier) domain of the hologram. This procedure limits the spatial frequency range that is effectively used for the reconstruction. This can be interpreted as a reduction of the angular range of the light that forms the real image and as a reduction of the effective numerical aperture. This generally leads to an increased particle-image depth-of-focus [6].

This paper proposes a simple, intuitive and effective method that suppresses the influence of the speckle pattern of the virtual image on DHPIV results without the above limitations: the reconstructed speckle pattern of the virtual image can be separated from the reconstructed real image by focusing on the different nature of the real image and the speckle pattern of the virtual image. With a suspended particle field as the object, the real image is composed of several strongly localized high-amplitude peaks, whereas the speckle pattern of the virtual image is a relatively low-amplitude field that is homogeneously distributed in the reconstruction volume. We therefore propose the intuitive solution to exclude, from further calculations, any pixels in the reconstructed volume that have an absolute light amplitude below a certain threshold. This is achieved by numerically setting all pixels where the absolute value of the light amplitude is smaller than a chosen threshold to zero. The amplitude threshold, $U_{t}$, is defined as the product of an appropriate threshold factor, $\zeta$, and the maximum absolute value of the complex light amplitude of the considered interrogation volume, $\left|U_{1,2}(x, y, z)\right|_{\max }$, where the indices ' 1 ' and ' 2 ' refer to the reconstructed particle field before and after displacement, respectively. The threshold factor, $\zeta$, can vary between 0 and 1 . The optimization of $\zeta$ is discussed at the end of this section:

$$
\begin{aligned}
& U_{t 1}=\zeta \cdot\left|U_{1}(x, y, z)\right|_{\max } \\
& U_{t 2}=\zeta \cdot\left|U_{2}(x, y, z)\right|_{\max } .
\end{aligned}
$$

Since the maximum light amplitude of interrogation volumes 1 and 2 may vary slightly, the amplitude threshold, $U_{t}$, of the two interrogation volumes may also be slightly different. This

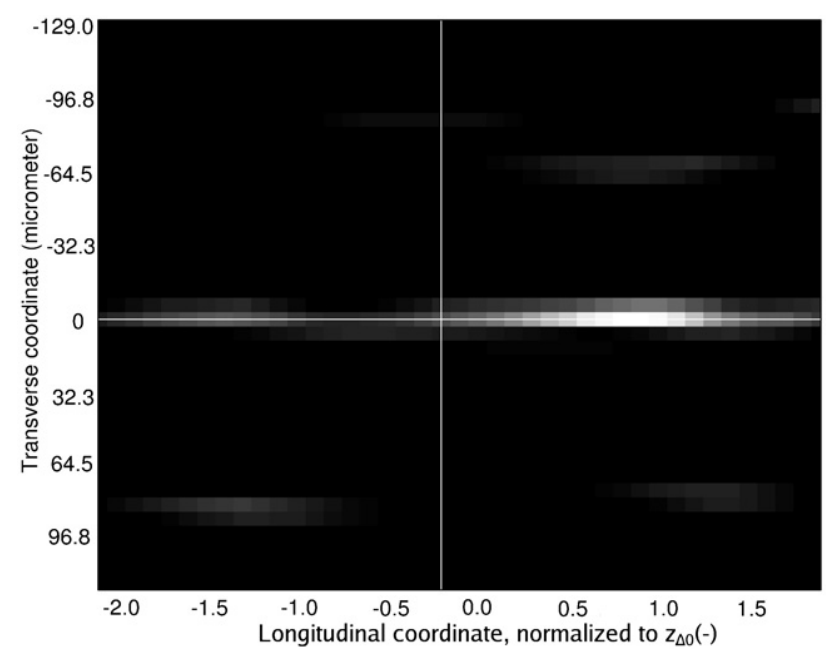

Figure 10. A slice through a correlation volume of an on-axis, experimentally recorded particle field that was translated $1 \mathrm{~mm}$ $\left(=z_{\Delta 0}\right)$ between the two recordings. A threshold factor of 0.6 was applied. The grey scale represents the correlation coefficient. The virtual-image peak that was present on the left (figure 1) has disappeared.

method does not only suppress the speckle pattern of the virtual image, but also the relatively weak out-of-focus component of the real image. It is expected that this further results in an accurate agreement between the local displacement of the recorded particles and the local measured particle field displacement. Furthermore, it is noted that the threshold operation is probably only effective if the real particle images have a similar brightness. A large variation of the particleimage brightness could lead to an unwanted discard of the weaker particle images by the threshold operation.

The threshold procedure is applied to the interrogation volumes described in section 4. Applying a threshold factor of 0.6 to the reconstructed amplitude leads to a correlation volume of which a slice is shown in figure 10 . The left (virtual-image) peak has nearly disappeared while the right (real-image) peak remains intact. This change suggests that the sign ambiguity (and possibly the bias error) of $z_{\Delta m}$ are suppressed. The histogram in figure 11 confirms this: the left peak for negative longitudinal displacement has completely disappeared, which illustrates that the peak-finding algorithm (section 3) mainly detects the real-image peak in the correlation volume. Additionally, the histogram peak for positive displacement is now centred around $z_{\Delta m} / z_{\Delta 0}=+1.0$ which illustrates that the bias error of $z_{\Delta m}$ is suppressed.

The effect of the threshold factor on the sign ambiguity of $z_{\Delta m}$ is shown by plotting a sign-ambiguity factor $(\Gamma)$ versus the threshold factor, $\zeta$ (figure 12). The factor $\Gamma$ is extracted from the 1000 correlation results and is defined as the number of detections of the virtual-image correlation peak $\left(=n_{v}\right)$ divided by the number of detections of the virtual-image peak or the real-image peak $\left(=n_{v}+n_{r}\right)$ or $\Gamma=n_{v} /\left(n_{v}+n_{r}\right)$. When $\Gamma=0.0$, the sign ambiguity is absent and when $\Gamma=0.5$, the sign ambiguity is most present. The factor $\Gamma$ can be larger than 0.5 as explained at the end of section 4 . In this experiment, a measured displacement is considered to correspond to the 


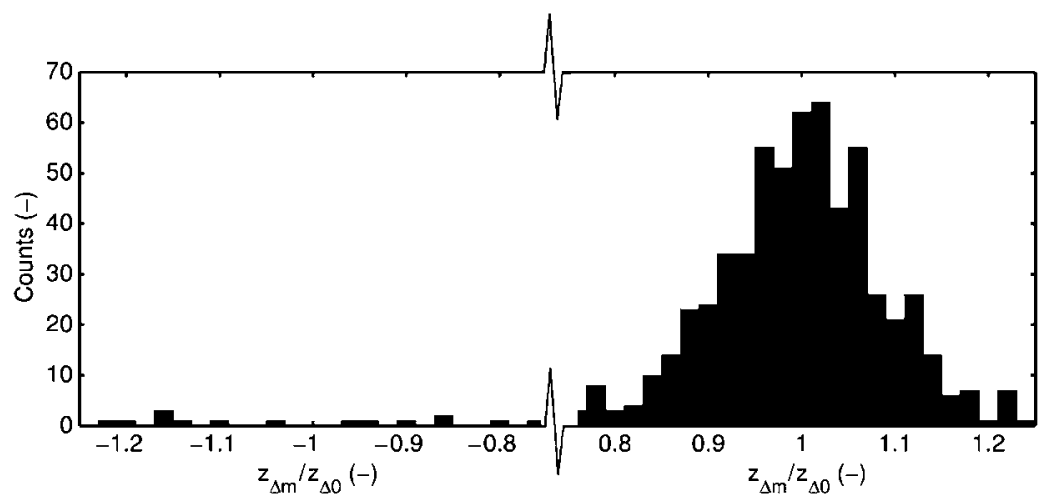

Figure 11. Histogram of the longitudinal component of the experimentally measured particle field displacement. One thousand interrogation volumes are analysed. A threshold factor of 0.6 is applied. Measurements with a very low correlation coefficient $\left(r_{12}<0.10\right)$ are excluded. The sign ambiguity of $z_{\Delta m}$ has practically disappeared.

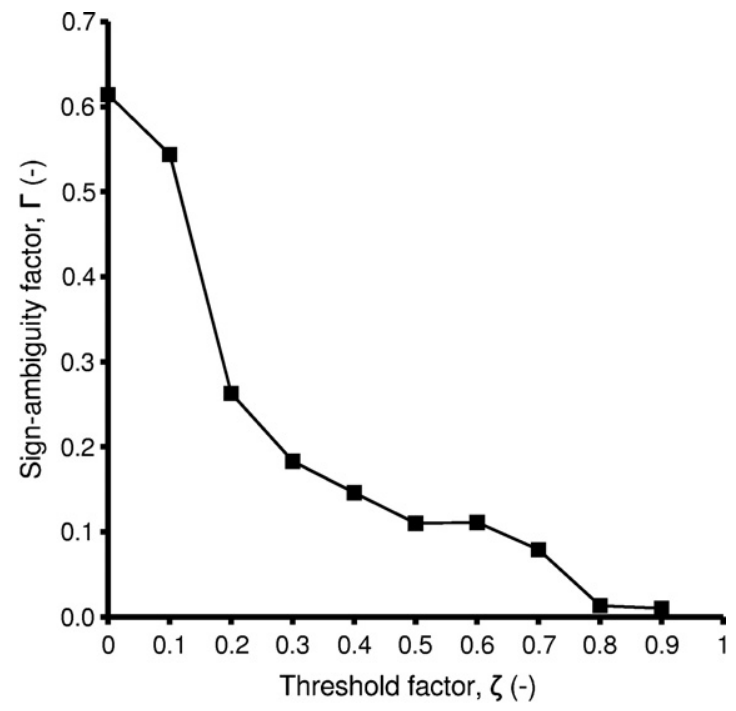

Figure 12. The sign-ambiguity factor (defined as $\Gamma=n_{v} /\left(n_{v}+n_{r}\right)$ ) is plotted against the applied threshold factor for the experimental results of section 4 . The sign ambiguity of $z_{\Delta m}$ clearly decreases for an increasing threshold factor. Data points at $\zeta=1.0$ are not calculated because this case implies that all pixels in the two interrogation volumes are set to zero.

real-image peak if the longitudinal displacement is between $+0.7 z_{\Delta 0}$ and $+1.3 z_{\Delta 0}$ and the transverse displacement is between $-20 \mu \mathrm{m}$ and $+20 \mu \mathrm{m}$. Similarly, the measured displacement is considered to correspond to the virtual-image peak when the longitudinal displacement is between $-1.3 z_{\Delta 0}$ and $-0.7 z_{\Delta 0}$. It is observed that the choice of these values practically does not influence final results. Figure 12 shows a clear suppression of the sign ambiguity of $z_{\Delta m}$ for an increasing threshold factor.

The effect of the threshold factor on the bias error is shown by plotting the mean measured longitudinal displacement (=the centre-of-gravity of the positive histogram peak) versus the threshold factor (figure 13). Again, only correlation results with a longitudinal displacement between $+0.7 z_{\Delta 0}$ and $+1.3 z_{\Delta 0}$ and a transverse displacement between $-20 \mu \mathrm{m}$ and $+20 \mu \mathrm{m}$ are included in the calculation of the centre-of-gravity.

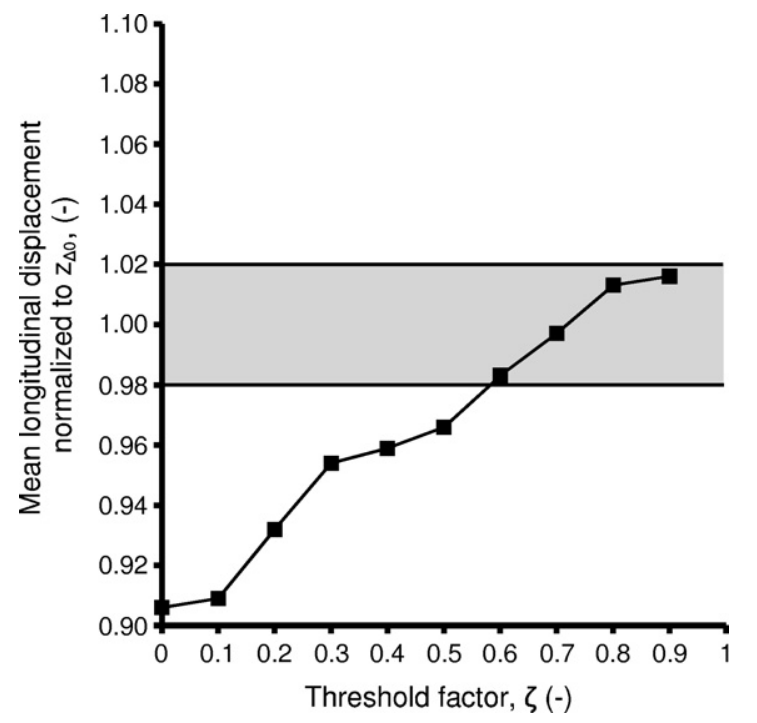

Figure 13. The centre-of-gravity of the histogram peak (figure 11), or mean longitudinal displacement, is plotted against the applied threshold factor. The bias error of $z_{\Delta m}$ is suppressed to less than $2 \%$ for larger threshold factors $(\zeta \geqslant 0.6)$.

Figure 13 shows that, without applying a threshold, the bias error of the measured longitudinal displacement is nearly $10 \%$ and that the bias error of the longitudinal shift is suppressed to less than $2 \%$ when the threshold factor is larger than 0.6.

The measured $z_{\Delta m}$ from individual correlation volumes has a noticeable inaccuracy, as can be seen by the width of the histogram peak in figure 11. The variance of the $1000 z_{\Delta m^{-}}$ values is calculated for different threshold factors. Again, only correlation results with a longitudinal displacement between $+0.7 z_{\Delta 0}$ and $+1.3 z_{\Delta 0}$ and a transverse component between $-20 \mu \mathrm{m}$ and $+20 \mu \mathrm{m}$ are included in the calculation. Figure 14 illustrates that the inaccuracy of individual measured longitudinal displacements increases for a larger threshold factor. The increase of the variance for an increasing threshold factor is caused by a reduced amount of pixels in an interrogation volume that contain the particle-image information. 


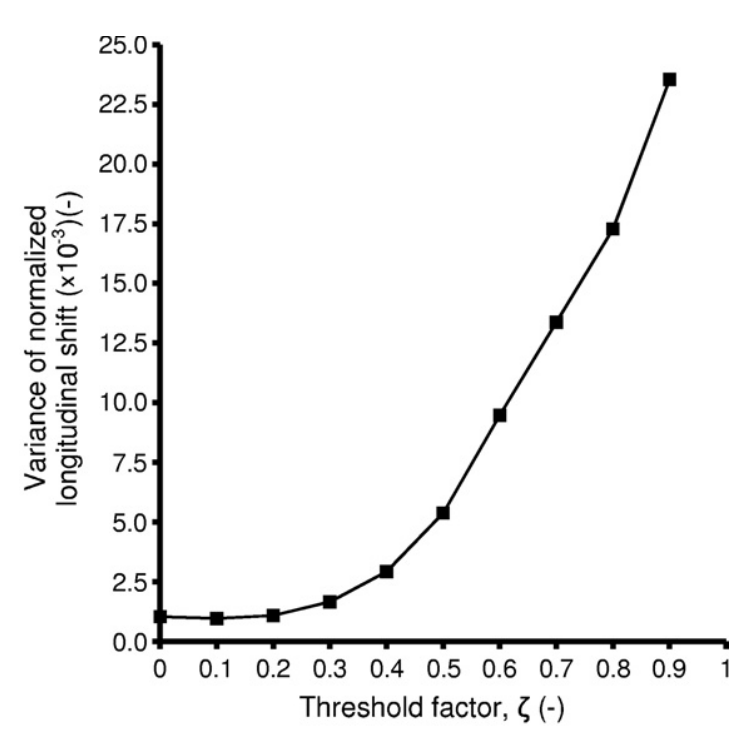

Figure 14. The variance of 1000 experimentally measured $z_{\Delta m}$-values versus the threshold factor. The variance is a measure for the width of the histogram peak in figure 11 and hence for the uncertainty of the measured longitudinal shift. The increase of the variance for an increasing threshold factor is caused by a decreasing amount of pixels in an interrogation volume that contains particle-image information.

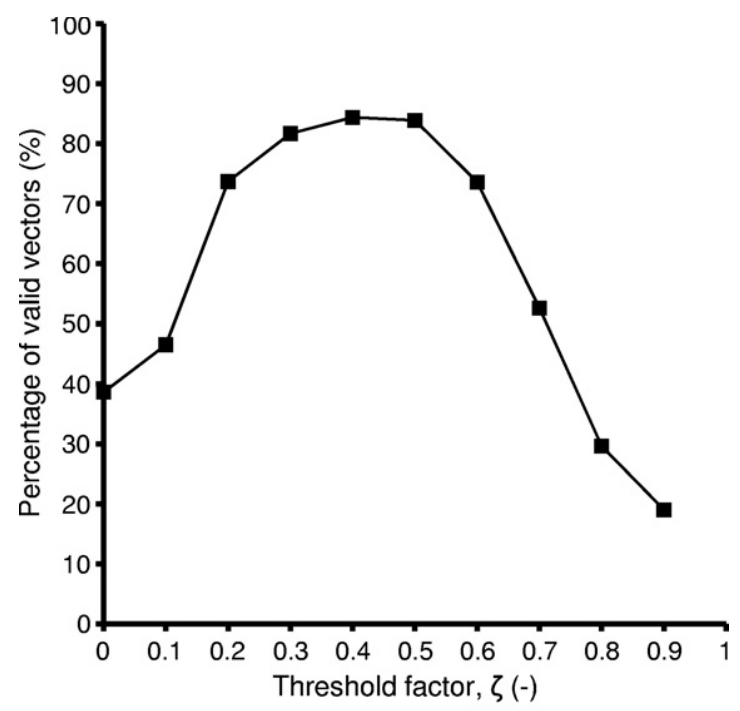

Figure 15. The number of valid displacement vectors versus the corresponding threshold factor. A vector is considered valid when it is sufficiently close to $\left(0,0,+z_{\Delta 0}\right)$. These data are based on the experimental results of section 4 .

The optimal value of the threshold factor is influenced by opposing factors. A larger threshold factor leads to a stronger suppression of sign ambiguity of $z_{\Delta m}$. However, a larger threshold factor also increases the inaccuracy of the individual measured longitudinal displacements, as explained in the previous paragraph. The combined effect is illustrated by evaluating the amount of valid vectors for different threshold factors, as illustrated in figure 15 . A vector is considered valid when it is sufficiently close to $\left(0,0,+z_{\Delta 0}\right)$, in other words when the longitudinal vector component is between $+0.7 z_{\Delta 0}$ and $+1.3 z_{\Delta 0}$ and when the transverse vector component is between $-20 \mu \mathrm{m}$ and $+20 \mu \mathrm{m}$. The curve in figure 15 shows an increase of valid vectors for small threshold factors, which is caused by the suppression of the sign ambiguity. The number of valid vectors decreases for higher threshold factors, which is caused by the higher inaccuracy of the individual measured longitudinal displacement. The largest amount of valid vectors is found at a threshold factor of 0.4 . It would be desirable that for this threshold factor, all 1000 vectors would qualify as a valid vector. The reason for the lower amount of valid vectors at the maximum of $84 \%$ is probably caused by the relatively low particle seeding. The values of $84 \%$ and $88 \%$ are higher than that predicted by simulations by Keane and Adrian [8] (55\%-80\%), but this can be explained by the contribution of the out-of-focus particle image to the correlation result and by the well-controlled pure uniform particle displacement (section 4).

\section{Conclusions and discussion}

In on-axis digital holographic PIV, the reconstruction volume contains an overlapping real image and speckle pattern of the virtual image. When the particle field experiences a longitudinal displacement between two recordings and when the two reconstructions are analysed with a 3D correlation analysis, two separate correlation peaks appear in the correlation volume at opposite $z$-positions. One peak is related to the displacement of the reconstructed real image while the other peak is related to the displacement of the reconstructed speckle pattern of the virtual image. It is shown in section 2 that both peaks have a comparable height when the reconstructed complex amplitude is used. This leads to a sign ambiguity in the longitudinal component of the measured particle field displacement, $z_{\Delta m}$. Additionally, the measured longitudinal particle field displacement suffers from a bias error. It is shown in appendix A that the bias error is caused by the presence of the speckle pattern of the virtual image in the reconstruction region. The real-image correlation peak is shifted towards a lower absolute $z$-coordinate by the presence of a correlation-coefficient gradient that originates from the virtual-image correlation peak.

It is shown in section 5 that the sign ambiguity and the bias error of $z_{\Delta m}$ can be suppressed by applying a threshold operation to the reconstructed amplitude in each interrogation volume. Experimental results illustrate that the threshold operation suppresses the sign ambiguity from $\Gamma=0.61$ when $\zeta=0$ to $\Gamma=0.01$ when $\zeta=0.8$. Also, the bias error is reduced from $10 \%$ to less than $2 \%$ when the threshold factor is sufficiently high $(\zeta \geqslant 0.6)$. On the other hand, the inaccuracy of the individual measured displacements is larger for larger threshold factors. These opposing trends result in a maximal amount of valid vectors for a threshold factor with an intermediate value ( $\zeta=0.4$ in this case). This experimental investigation has been conducted with particles with a comparable diameter $( \pm 20 \%)$. A larger variation of the particle diameter could lead to a large variation of the particleimage brightness. In that case, the threshold operation should 
be monitored closely to minimize unwanted discards of weaker particle images.

\section{Acknowledgments}

We would like to thank the FOM Foundation for the financial support, Wilco Tax for the technical support and Joseph Braat, Koh Ikeda and Christian Poelma for the useful discussions.

\section{Appendix. Cause of the bias error of the measured $z_{\Delta}$}

\section{A.1. Theoretical analysis}

As described in section 1, the average measured longitudinal displacement of a recorded particle field differs from the applied nominal displacement, $z_{\Delta 0}$. This effect is also observed in experimental results (figure 8). Although the expected position of the right histogram peak is at $z_{\Delta m} / z_{\Delta 0}=$ +1 , the peak is centred at a lower $z_{\Delta m}$-value, in this case near $z_{\Delta m}=0.9 z_{\Delta 0}$. The cause of this bias error is investigated in this section. The investigation starts with the hypothesis that the presence of the virtual-image peak near the realimage peak creates the bias error. The virtual-image peak in the correlation volume introduces a correlation-coefficient gradient $(\mathrm{d} r / \mathrm{d} z)$ at the position of the real-image peak. This gradient of the virtual-image peak is superimposed on the real-image peak, which leads to a shift of the position of the maximum of the real-image peak, which implies a disturbance of the measured longitudinal displacement. This effect is relatively strong: the low numerical aperture of a digital holographic set-up leads to reconstructed particles images that are stretched along the longitudinal direction. The stretched shape of the particle images reappears in the shape of the realimage peak and the virtual-image peak. The stretched virtualimage peak generates substantial gradients at a relatively large longitudinal distance. Because both peaks are located at the same $x$ - and $y$-coordinates, the gradient of the virtual-image peak may be substantial at the position of the real-image peak. Additionally, the stretched shape of the real-image peak makes it relatively sensitive to additional gradients near its maximum. A mathematical analysis is presented that leads to a relation between the height of the virtual-image peak, the height of the real-image peak and the expected longitudinal displacement of the maximum of the real-image peak (bias error). Then, the obtained relation is compared to results of numerical simulations and experiments to investigate whether the above hypothesis correctly describes the mechanism that leads to the bias error.

The longitudinal profile of the real-image correlation peak is written as a product of its height $r_{r}$ and a dimensionless function $f_{r}(z)$ that describes the longitudinal profile through the maximum of the peak:

$$
r\left(x_{\Delta 0}, y_{\Delta 0}, z\right)=r_{r} \cdot f_{r}(z) .
$$

At the maximum of the unaffected peak $\left(z_{\Delta}=z_{\Delta 0}\right)$, the profile is approximated by a second-order Taylor polynomial:

$$
r\left(x_{\Delta 0}, y_{\Delta 0}, z\right) \approx r_{r} \cdot\left(1-\left(\frac{z-z_{\Delta 0}}{\sigma}\right)^{2}\right)
$$

where $\sigma$ is a measure for the width of the peak. When a disturbing gradient $p$ is added in the region of the maximum, the position of the maximum moves from $z_{\Delta 0}$ to

$$
z_{\Delta 0}+\frac{p \cdot \sigma^{2}}{2 \cdot r_{r}}
$$

The second term of equation (A.3) is considered the bias error.

The profile of the virtual-image peak on the longitudinal line through its maximum is written as a product of the height of the peak $r_{v}$ and a dimensionless function $f_{v}(z)$ :

$$
r\left(x_{\Delta 0}, y_{\Delta 0}, z\right)=r_{v} \cdot f_{v}(z) .
$$

The derivative of the height of the virtual-image correlation peak to $z$ at the position of the real-image peak is

$$
\frac{\mathrm{d} r}{\mathrm{~d} z}\left(x_{\Delta 0}, y_{\Delta 0},+z_{\Delta 0}\right)=r_{v} \cdot \frac{\mathrm{d} f_{v}\left(+z_{\Delta 0}\right)}{\mathrm{d} z} \text {. }
$$

Substituting $\mathrm{d} r / \mathrm{d} z\left(x_{\Delta 0}, y_{\Delta 0},+z_{\Delta 0}\right)$ of equation (A.5) into the variable $p$ of equation (A.3) yields that the bias error is proportional to the ratio of the height of the virtual-image correlation peak and the real-image correlation peak:

$$
\text { bias_error } \propto \frac{r_{v}}{r_{r}} \text {. }
$$

\section{A.2. Numerical simulation}

In the model, four reconstruction volumes are defined, R1, R2, $\mathrm{V} 1$ and $\mathrm{V} 2$, where ' $\mathrm{R}$ ' indicates a real image, ' $\mathrm{V}$ ' indicates a speckle pattern that originates from the virtual image and ' 1 ' and ' 2 ' indicate a particle-image field before and after displacement. By separately defining the real image and the speckle pattern of the virtual image, any superposition of R1 and V1 can be correlated with an equal superposition of R2 and V2. With this method, the contribution of the speckle pattern of the virtual image to the interrogation can be varied, and its effect on the position of the real-image peak can be investigated. This is not possible with the numerical model that is used in section 3 where the energy in the real image and the energy in the speckle pattern of the virtual image are always equal.

Volume $\mathrm{R} 1$ contains a real particle image located at $\left(x_{p}, y_{p}\right.$, $\left.z_{p}\right)$. Volume R2 contains a real particle image located at ( $x_{p}$, $\left.y_{p}, z_{p}+z_{\Delta 0}\right)$. The reconstructed particle image is modelled as a cone with an opening angle that corresponds to the NA of our experimental set-up. Because the $3 \mathrm{D}$ speckle pattern in $\mathrm{V} 1$ is a collection of many diffraction-limited point-source images [7], the $3 \mathrm{D}$ speckle pattern is modelled by a superposition of several (100) real particle images with a reduced intensity. Volume $\mathrm{V} 2$ contains the field of $\mathrm{V} 1$, shifted $\left(0,0,-z_{\Delta 0}\right)$. The field $(\mathrm{R} 1+\kappa \cdot \mathrm{V} 1)$ is correlated with $(\mathrm{R} 2+\kappa \cdot \mathrm{V} 2)$, where the constant $\kappa$ allows the selection of any superposition of the real image and the speckle pattern of the virtual image. Consequently, variation of $\kappa$ implies a variation of the ratio of the height of the virtual-image correlation peak and the height of the real-image correlation peak $\left(r_{v} / r_{r}\right)$. The numerical simulation analyses the bias error $\left(z_{\Delta m}-z_{\Delta 0}\right)$ for different values of $\kappa$ and $z_{\Delta 0}$. The bias error is expected to be proportional to $r_{v} / r_{r}$ (equation (A.6)). Further, it is expected that when $z_{\Delta 0}$ is large, and the two correlation peaks are widely separated, the slope 


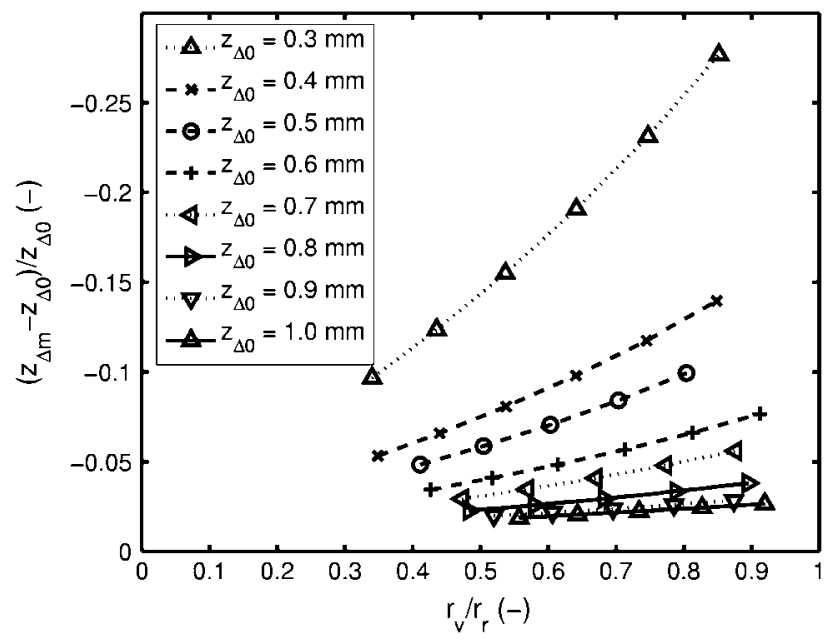

Figure 16. The dimensionless bias error $\left(\left(z_{\Delta m}-z_{\Delta 0}\right) / z_{\Delta 0}\right)$ is plotted versus the ratio of the height of the virtual-image peak and the real-image peak $\left(r_{v} / r_{r}\right)$ for various nominal $z$-displacements. This result follows from numerical simulations.

of the virtual-image peak at the position of the maximum of the real-image peak is smaller. This is expected to lead to a small bias error.

In figure 16, the bias error is plotted versus $r_{v} / r_{r}$ for various $z_{\Delta 0}$. The bias error is divided by the nominal $z$ displacement to obtain a dimensionless bias error $\left(\left(z_{\Delta m}-\right.\right.$ $\left.\left.z_{\Delta 0}\right) / z_{\Delta 0}\right)$. As expected, the bias error and $r_{v} / r_{r}$ show a fair linear relationship for each value of $z_{\Delta 0}$. The dimensionless as well as the absolute bias error decreases for an increasing $z_{\Delta 0}$.

\section{A.3. Experimental study}

The experimental results of section 4 are analysed to see whether a linear relationship between the bias error and $r_{v} / r_{r}$ exists. For each of the 1000 interrogations, $r_{r}, r_{v}$ and the bias error are determined. Because the uncertainty of $r_{r}, r_{v}$ and the bias error of the individual interrogations is quite large, the data are grouped. The dataset is sorted according to ascending $r_{v} / r_{r}$-value and then five groups of 200 original points are formed. The resulting 5 points are plotted in figure 17, where the horizontal position of the 5 points is the average of the $r_{v} / r_{r^{-}}$ values of the grouped 200 points and the vertical position is the average of the bias-error values of the grouped 200 points. The error bar indicates the $95 \%$ confidence interval, $\delta_{95}$, and is given by Chatfield [28] as

$$
\delta_{95}=t_{c} \frac{s}{\sqrt{n}}
$$

where $t_{c}$ describes the $t$-distribution statistics for a $95 \%$ confidence interval, $s$ is the sample standard deviation and $n$ is the sample size. In this case, $n$ equals 200 which leads to $t_{c}$ equals 1.96 [28]. The variable $s$ is the sample standard deviation of the $r_{v} / r_{r}$-values and the bias-error values of the grouped 200 points. A linear least-squares fit (model: $y=$ $a x+b)$ is performed on the original 1000 data points and

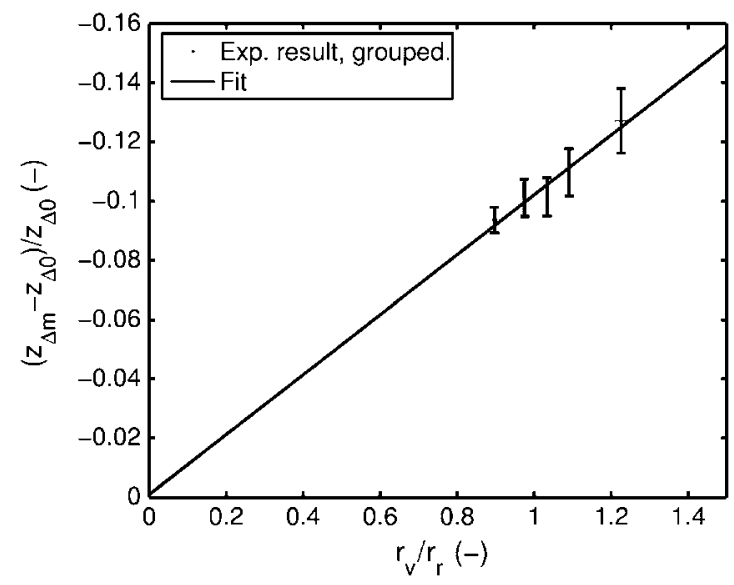

Figure 17. The dimensionless bias error $\left(\left(z_{\Delta m}-z_{\Delta 0}\right) / z_{\Delta 0}\right)$ is plotted versus the ratio of the height of the virtual-image peak and the real-image peak $\left(r_{v} / r_{r}\right)$. The five data points are obtained by creating and averaging five groups of 200 data points. This result follows from experimental data.

extrapolated to $r_{v} / r_{r}=0$ to check whether a linear relationship $(y=a x, b=0)$ exists. Figure 17 clearly shows that the fit crosses the axes near the origin. Although strong averaging of the original data points and a long extrapolation is needed, a clear linear relationship between the bias error and $r_{v} / r_{r}$ is observed.

Summarizing, a hypothesis and a theoretical model suggest that the bias error originates from a longitudinal shift of the real-image peak which is caused by a correlationcoefficient gradient $(\mathrm{d} r / \mathrm{d} z)$ that originates from the virtualimage peak. This mechanism predicts a linear relationship between the bias error and $r_{v} / r_{r}$. This linear relationship is indeed observed in numerical simulation results and experimental results. It is also observed in the numerical simulation results that the bias error decreases with an increasing $z_{\Delta 0}$, which is in agreement with the hypothesis. It is concluded that the hypothesis correctly describes the mechanism that leads to the bias error. In other words, the presence of the speckle pattern of the virtual image in the reconstruction region and the inherent presence of the virtual-image peak in the correlation volume are identified as the cause of the bias error.

\section{References}

[1] Adams M, Kreis T M and Jüptner W P O 1997 Particle size and position measurement with digital holography Optical Inspection and Micromeasurements: II. Proc. SPIE vol 3098, ed C Gorecki, pp 234-40

[2] Murata S and Yasuda N 2000 Potential of digital holography in particle measurement Opt. Laser Technol. 32 567-74

[3] Pan G and Meng H 2003 Digital holography of particle fields: reconstruction using complex amplitude Appl. Opt. 42 827-33

[4] Malkiel E, Sheng J, Katz J and Strickler J R 2003 The 3-dimensional flow field generated by a feeding calanoid copepod measured using digital holography J. Exp. Biol. $2063657-66$ 
[5] Ikeda K, Okamoto K and Murata S 2003 Development of dynamic digital holographic particle velocimetry 5 th Int. Symp. on Particle Image Velocimetry (Busan, Korea, 22-24 September 2003) ed S J Lee et al p 3103

[6] Ooms T A, Koek W D, Braat J J M and Westerweel J 2006 Optimizing Fourier filtering for digital holographic particle image velocimetry Meas. Sci. Technol. 17 304-12

[7] Goodman J W 1996 Introduction to Fourier Optics ed S W Director et al (Singapore: McGraw-Hill)

[8] Keane R D and Adrian R J 1992 Theory of cross-correlation analysis of PIV images Appl. Sci. Res. 49 191-215

[9] Barnhart D H, Adrian R J and Papen G C 1994 Phase-conjugate holographic system for high-resolution particle-image-velocimetry Appl. Opt. 33 7159-70

[10] Pu Y and Meng H 2000 An advanced off-axis holographic particle image velocimetry (PIV) system Exp. Fluids 29 184-97

[11] Sheng J, Malkiel E and Katz J 2003 Single beam two-views holographic particle image velocimetry Appl. Opt. 42 235-50

[12] Hinsch K D and Herrmann S F 2004 Signal quality improvements by short-coherence holographic particle image velocimetry Meas. Sci. Technol. 15 622-30

[13] Chan V S S, Koek W D, Barnhart D H, Bhattacharya N, Braat J J M and Westerweel J 2004 Application of holography to fluid flow measurements using bacteriorhodopsin (bR) Meas. Sci. Technol. 15 647-55

[14] Vikram C S 1992 Particle Field Holography ed P L Knight and W J Firth (Cambridge: Cambridge University Press)

[15] Cuche E, Marquet P and Despeursinge C 2000 Spatial filtering for zero-order and twin-image elimination in digital off-axis holography Appl. Opt. 39 4070-5

[16] Raffel M, Willert C and Kompenhans J 1998 Particle Image Velocimetry: A Practical Guide ed R J Adrian et al (Berlin: Springer)
[17] Coupland J M and Halliwell N A 1997 Holographic displacement measurements in fluid and solid mechanics: immunity to aberrations by optical correlation processing Proc. R. Soc. A 453 1053-66

[18] Shanmugan K S and Breipohl A M 1988 Random Signals: Detection, Estimation and Data Analysis (New York: Wiley)

[19] Koek W D, Bhattacharya N, Ooms T A, Westerweel J and Braat J J M 2005 Influence of virtual images on the signal-to-noise ratio in digital in-line particle holography Opt. Express $132578-89$

[20] Adrian R J 1986 Image shifting technique to resolve directional ambiguity in double pulsed velocimetry Appl. Opt. 25 3855-8

[21] Coupland J M 2004 Holographic particle image velocimetry: signal recovery from under-sampled CCD data Meas. Sci. Technol. 15 711-7

[22] Westerweel J 1997 Fundamentals of digital particle image velocimetry Meas. Sci. Technol. 8 1379-92

[23] Yang H, Halliwell N and Coupland J M 2003 Digital shearing method for three-dimensional data extraction in holographic particle image velocimetry Appl. Opt. 42 6458-64

[24] Herrmann S F and Hinsch K D 2004 Light-in-flight holographic particle image velocimetry for wind-tunnel applications Meas. Sci. Technol. 15 613-21

[25] Meng H, Pan G, Pu Y and Woodward S H 2004 Holographic particle image velocimetry: from film to digital recording Meas. Sci. Technol. 15 673-85

[26] Onural L and Scott P D 1987 Digital decoding of in-line holograms Opt. Eng. 26 1124-32

[27] Schnars U and Jüptner W 1994 Direct recording of holograms by a CCD target and numerical reconstruction Appl. Opt. 33 179-81

[28] Chatfield C 1970 Statistics for Technology (London: Chapman and Hall) 